\title{
Enhanced Matching and Vialess Decoupling of Nearby Patch Antennas for MIMO System
}

\author{
Amirhossein Alizadeh Ghannad, Student Member IEEE, Mohsen Khalily, Senior Member IEEE, \\ Pei Xiao, Senior Member IEEE, Rahim Tafazolli, Senior Member IEEE, and Ahmed A. Kishk, Fellow IEEE
}

\begin{abstract}
Simultaneous improvement of matching and isolation for a modified two-element microstrip patch antenna array is proposed. Two simple patch antennas in a linear array structure are designed, whereas, the impedance matching and isolation are improved without using any conventional matching networks. The presented low profile multifunctional via-less structure comprises of only two narrow $T$-shaped stubs connected to feed lines, a narrow rectangular stub between them, and a narrow rectangular slot on the ground plane. This design provides a simple, compact structure with low mutual coupling, low cost and no adverse effects on the radiation and resonance. To validate the design, a compact very-closely-spaced antenna array prototype is fabricated at $5.5 \mathrm{GHz}$ which is suitable for multiple-input-multiple-output (MIMO) systems. The measured and simulated results are in good agreement with a $16 \mathrm{~dB}$, and $40 \mathrm{~dB}$ of improvements in the matching and isolation, respectively.
\end{abstract}

Index Terms-Multiple-input and multiple output (MIMO), array antenna, isolation, matching.

\section{INTRODUCTION}

Different communication technologies, such as phased array antennas and radars, various smart arrays, and the massive multipleinput-multiple-output (M-MIMO) technique, are the enablers for different current and future military and civilian applications $[1,2]$. To design these array structures in microstrip technology, we need different feeding techniques in the first step to improve the impedance characteristics of the antennas under the influence of the other array elements. In general, the input impedance of an antenna is different from the impedance of the system to which it is connected [3]. In many cases, impedance matching can be improved by modifying the antenna geometry [3-5]. As an example, in [4], a broad-band matching and stable radiation characteristics are achieved within about $13 \mathrm{GHz}$ centered at $8 \mathrm{GHz}$ by employing three slots in two corners of the square patch. In $50-\Omega$ microstrip-fed line patch antennas, there are various feeding techniques available for impedance matching, such as recessed-line (inset-feed), quarter-wave transformer, displaced lines, etc. $[3,4]$. Using these techniques, the patch structure is compromised, or the size of feed lines increases. Therefore, these methods are not suitable for modern dense array systems. Therefore, we need a new matching structure to fit within the array structure without adding extra space.

In addition to impedance matching in the compact arrays, these systems are likely to suffer from a high degree of mutual coupling between array elements [6, 7]. As the need for compact arrays is growing, the full size of the array system becomes smaller. As a result, the performance of the array without the isolators is noticeably degraded due to the weak isolation between adjacent elements [1, 2, 8]. According to the recent studies, poor isolation between the array elements leads to high correlation and has an adverse effect on the capacity of a MIMO system. In addition, it reduces the output signalto-noise ratio, the efficiency of an adaptive array antenna, and the convergence of array signal processing algorithms [1, 2, 7-9]. There- fore, the reduction of the mutual coupling is essential for MIMO.

In recent years, a variety of techniques including different shapes of defected ground structures (DGS) [9, 10-13], parasitic elements [2, $8,14,15]$, electromagnetic band-gap structures [16], polarization conversion isolators [12], and metamaterial-based DGSs [13] have been reported to improve the isolation in MIMO array system. An indepth study of these techniques shows some of their restrictions, including the need for larger size and multi-layer structure and high design and fabrication complexity and cost [1, 2, 8-16].

A large-scale array antenna was recently proposed in [1] with considerably reduced mutual coupling using an array-antenna decoupling surface on top of the antennas. A simple and efficient parasitic isolator was proposed in [2] without DGS and with more than $50 \mathrm{~dB}$ isolation for a two-element array with an edge-to-edge distance of about $0.06 \lambda_{0}$ (where $\lambda_{0}$ is the free space wavelength of the resonance). A new method provided by employing a pixel-type parasitic isolator between the antennas was presented in [12] to adjust the polarization of the coupling fields between the antennas. A metamaterial-based isolator was used in [13] to improve the isolation at each band of a dual-band MIMO system. In this system, a mutual coupling reduction of more than $20 \mathrm{~dB}$ at each band has been achieved. A comprehensive review of various mutual coupling reduction techniques can be found in [17].

Recently, two works including both decoupling and matching networks (DMN), simultaneously as a network, were presented for two asymmetric [18] and symmetric [19] array antennas along with good matrix-based studies. In [18], a two layer DMN network with via, some connections between the feed lines and an increased board area was proposed. In [19], a simple DMN network was firstly defined by lumped elements and then distributed to microstrip lines. This via-less design increases the total board size, considerably.

In this paper, a new complex feeding network is designed for a twoelement microstrip-fed array antenna with a complete ground plane and without any conventional matching circuit to provide two following targets simultaneously: i) to significantly improve the impedance matching; and ii) to improve the isolation (the reduction of mutual coupling) between the two proposed antennas. The proposed low profile feeding circuit has two T-shaped stubs, a parasitic-based microstrip line between them and a narrow slot on the ground plane. The key parameters of the design are systematically studied and simulated, and their effects examined and discussed.

In the following, the design and simulation results are presented. Finally, the measured results, their discussions and also two critical parameters of the MIMO systems, including the Envelope Correlation Coefficient (ECC) and Diversity Gain (DG) are calculated.

It is also note that the Release 15 NSA defined two broad-spectrum ranges at sub- $6 \mathrm{GHz}$ and mmWave frequencies. To further support more potential sub- $6 \mathrm{GHz}$ frequency bands, an unlicensed LTE band namely as LTE band $46(5150-5925 \mathrm{MHz})$ is also considered for 5G massive MIMO antenna design [20]. The proposed decoupling and matching method has been designed and the prototype practically tested at $5.5 \mathrm{GHz}$ which can be suitable for the future 5G Massive MIMO application. In addition, the method has been verified at other $5 \mathrm{G}$ frequency bands such as Sub-6GHz, 3.6 GHz and mmWave 26 $\mathrm{GHz}$ but is not shown here for brevity. 


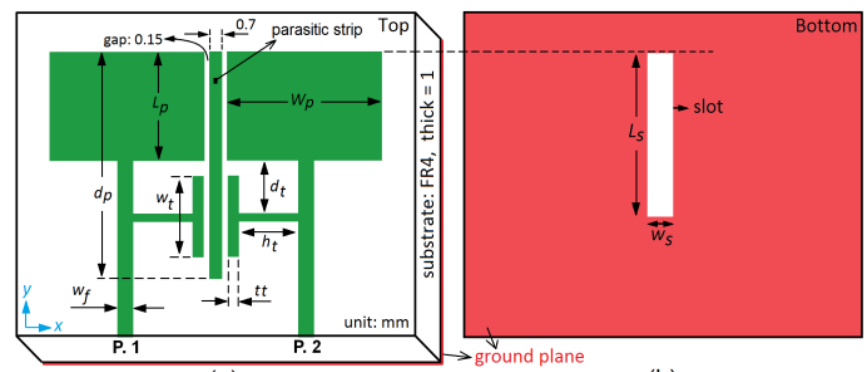

(a)

(b)

Fig. 1. The configuration of the proposed array antenna, (a) Top surface, and (b) Bottom surface of the substrate.

Table I. The optimal values of the parameters in the final design

\begin{tabular}{|c|c|c|c|c|c|c|}
\hline Parameter: & $d p$ & $W_{t}$ & $h_{t}$ & $t t$ & $L_{S}$ & $W_{S}$ \\
\hline $\begin{array}{l}\text { Values } \\
(\mathrm{mm}) \text { : }\end{array}$ & $\begin{array}{c}21.55 \\
\approx \\
0.39 \lambda_{0}\end{array}$ & $\begin{array}{c}6 \\
\approx \\
0.11 \lambda_{0}\end{array}$ & $\begin{array}{c}5.95 \\
\approx \\
0.108 \lambda_{0}\end{array}$ & 0.7 & $\begin{array}{c}16.55 \\
\approx \\
0.3 \lambda_{0}\end{array}$ & 1.8 \\
\hline
\end{tabular}

\section{Array Antenna Configuration, Design and Performance}

Fig. 1 shows the proposed microstrip-based patch antenna array. This antenna is designed on a printing FR4-based board with a relative permittivity of 4.4 , a loss tangent of 0.02 , and a thickness of $1 \mathrm{~mm}$. The total dimension of the substrate is $40 \mathrm{~mm}(x$-axis $) \times 32 \mathrm{~mm}$ ( $y$-axis) which is equal to $0.73 \lambda_{0} \times 0.55 \lambda_{0}$, where $\lambda_{0}$ is the free-space wavelength at the design frequency $(5.5 \mathrm{GHz})$. It should be noted that the main area that occupies the two antennas, matching and isolation structure, and two feeding lines are about $33 \mathrm{~mm} \times 22 \mathrm{~mm}$, which is about $0.6 \lambda_{0}$ $\times 0.4 \lambda_{0}$ as shown in Fig. 1. This area is smaller than those of the recently published works. The basic parameters, $W_{p}, L_{p}, W_{f}$, and $d_{t}$ are $16,12.55,1.9$ and $4.25 \mathrm{~mm}$, respectively. These parameters are constant in the simulation. The key parameters of the proposed matching and isolation structure are $d p, W_{t}, h_{t}, t t$ on the top surface and $L_{S}$ and $W_{S}$ of the slot, inserted on the ground plane (Fig. 1(b)). The optimal values of the parameters are summarized in Table I.

In the proposed array, the edge-to-edge distance between the patches is $1 \mathrm{~mm}$ (i.e., $0.018 \lambda_{0}$ at $5.5 \mathrm{GHz}$ ). This means that the proposed array with minimum distance can be a suitable sub-array of the larger arrays in novel systems such as massive MIMO. The centerto-center distance is $17 \mathrm{~mm}\left(0.31 \lambda_{0}\right)$ that is shorter $(38 \%)$ than the distance in the conventional array (i.e., $0.5 \lambda$ ). It should also be stated that when the distance between the center-to-center distance between the two patches is less than a half wavelength, the H-plane coupling (present case) is much higher than the E-plane one.

The proposed matching and isolation structure has two important roles: i) the improvement of the matching using a T-shaped stub with a variable impedance and electrical length parallel to the impedance of the feed line toward the patch; ii) the reduction of the mutual coupling using a band-notch filter consists of two T-shaped strips, a parasitic strip between them and a slot on the ground plane.

\section{Simulation AND PARAmetric StUdy}

In this section, first, six specific structures (specified by A to $F$, and proposed in the table in Fig. 2), as subsets of the proposed structure are considered and simulated to examine the performance of each subsection, individually. In this figure, the results of the proposed design with optimal parameters (Table I) and initial design (as a reference) without the matching and isolation structures are presented.

Fig. 2 shows the S-parameters of the initial structure in different cases that lead to the proposed structure. This figure indicates the matching $(6 \mathrm{~dB})$ and isolation $(8 \mathrm{~dB})$ between the two ports of the initial structures, which are not acceptable for the MIMO system. The solid lines in Fig. 2, which are corresponding to the proposed design,

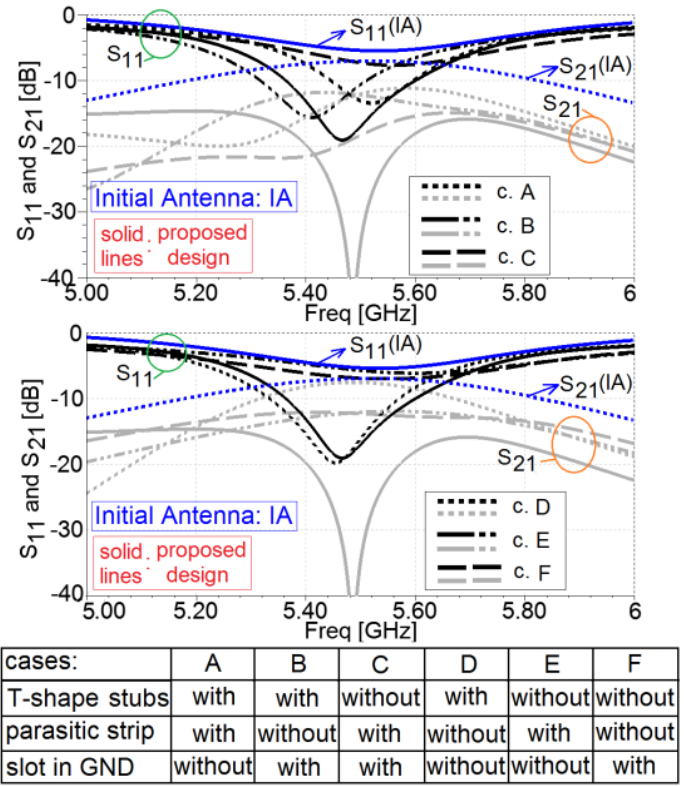

Fig. 2. The $S_{11}$ and $S_{21}$ graphs for six specific cases (A-F), in comparison with the proposed optimal array-antenna design and initial array antenna.

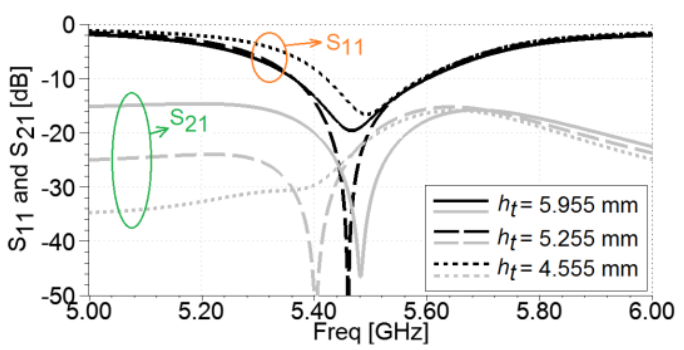

Fig. 3. The simulated $S_{11}$ and $S_{21}$ graphs of the proposed design for different values of $h_{t}$.

show that the matching and isolation are improved by approximately $15 \mathrm{~dB}$ and $33 \mathrm{~dB}$ around $5.5 \mathrm{GHz}$, respectively compared to the initial design (solid blue line). As a result, the proposed design can be a good choice for a MIMO system. Although, significant parameters of the MIMO system, ECC and DG must be determined to clarify the performance of the proposed design (compared to an initial array) in a MIMO standard. The results of case $\mathrm{C}$ show that the isolation of the design using parasitic strip and slot in the ground plane can be acceptable (about $20 \mathrm{~dB}$ ) around $5.5 \mathrm{GHz}$. However, the matching is degraded (by about $8 \mathrm{~dB}$ ). Therefore, these two parts affect the isolation, considerably. On the other hand, the T-shape stubs connected to the feed lines can effectively control the matching. These results can be verified by considering $S_{21}$ and $S_{11}$ of case $D$, which show that by using the T-shape stubs without the parasitic strip and slot, the matching is improved by about $16 \mathrm{~dB}$. However, the isolation is weak. The results in cases, $\mathrm{E}$ and $\mathrm{F}$ clarify that use of the parasitic strip or only the slot in the ground plane does not have a significant impact on the matching and isolation.

All parametric studies show that only four parameters, $d_{P}, L_{S}, W_{t}$, and $h_{t}$, can affect the matching and isolation. The first parameter that can affect the electrical length of the T-shaped stub is $h_{t}$. The related S-parameters are shown in Fig. 3. As illustrated, the matching of the resonant frequency can be adjusted considerably by controlling $h_{t}$.

In these cases, we can't see any significant changes in the isolation. The best choice of $h_{t}$ is $5.955 \mathrm{~mm}$ while the matching is about $19 \mathrm{~dB}$. The second key parameter, $W_{t}$, can affect both the stub electrical length and the coupling between the T-shape stubs and the parasitic strip, simultaneously.

Fig. 4 shows the results for different values of this parameter. By selecting $W_{t}$ around $2.75 \mathrm{~mm}$, the input impedance matching is about $19 \mathrm{~dB}$. The best isolation (about $53 \mathrm{~dB}$ ) and matching and the widest 


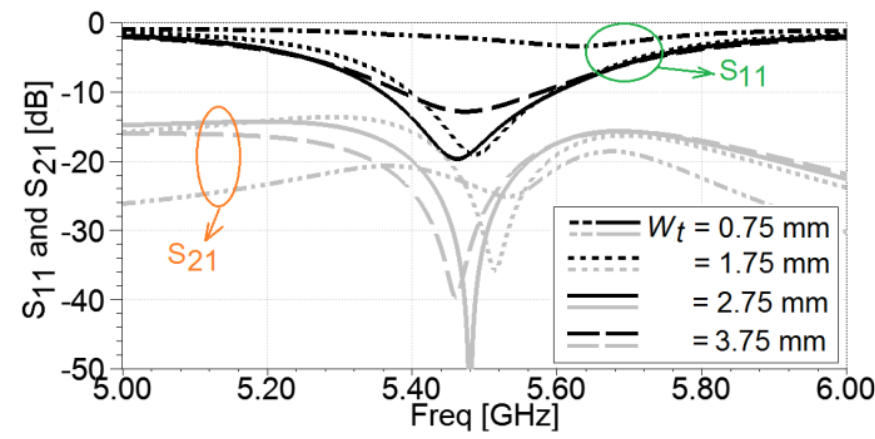

Fig. 4. Simulated $S_{11}$ and $S_{21}$ graphs of the design for different values of $W_{t}$.

10-dB $\mathrm{S}_{11}$ bandwidth $(5.33 \sim 5.62 \mathrm{GHz}, 5.3 \%)$ can be provided by setting $W_{t}$ equal to $2.75 \mathrm{~mm}$.

The next parameter is $t t$ (see Fig. 1). By increasing $t t$ from 0.2 to $1.5 \mathrm{~mm}$, the matching is degraded by about $5 \mathrm{~dB}$ around $5.5 \mathrm{GHz}$, and the isolation is improved from 35 to $45 \mathrm{~dB}$. In addition, the frequency, related to the largest value of $\left|S_{21}\right|$ is varied $0.05 \mathrm{GHz}$ around $5.5 \mathrm{GHz}$. These descriptions show that $t t$ has a small effect on the matching and isolation and then the related graphs are not shown here for brevity. The best choice for $t t$ is $0.7 \mathrm{~mm}$ in this design.

Two other key parameters are $d p$ and $L_{S}$, (illustrated in Figs. 1(a) and (b)) that the related S parameters are shown in Figs. 5(a) and (b). Fig. 5(a) shows that the changes in $d p$ have a little effect on the matching and position of the resonance. In these cases, the matching is acceptable and about $18.5 \mathrm{~dB}$. In addition, by setting $d p$ to $21.57 \mathrm{~mm}$ (the optimal value), very good isolation can be obtained about $43 \mathrm{~dB}$. The other values of $d p$ achieve about $15 \mathrm{~dB}$ around 5.5 GHz. These values of isolations cannot be adequate for MIMO system. On the other hand, the $L s$ has a significant impact on the resonance position and especially the level of the matching. As seen from Fig. 5(b), the best value of $L_{S}$ is $16.55 \mathrm{~mm}$.

The last parameter is $W_{S}$. Although, with $W_{S}$ around $1.8 \mathrm{~mm}$, the matching and isolation are varied around 19 and $40 \mathrm{~dB}$, respectively, this parameter has a small effect on the matching and isolation. Therefore, its results are not presented here for brevity. The structure can be scaled to cater for different frequencies. Therefore, these values are better to be expressed in terms of wavelength as in Table I.

To further clarify the isolation performance of the proposed design and present the blocking level of the coupling between two antennas, the E-field distributions before and after using the isolator are shown in Figs. 6(a) and (b), respectively. In this study, Port 1 (P.1) is excited and the second port (P.2) is matched by the $50-\Omega$ load. After employing the stubs and the parasitic strip and the slot, the field amplitude around the feed line of the second antenna and both radiating and non-radiating edges of the second patch are considerably reduced. Moreover, a strong field is induced on the left stub and the parasitic strip. This means that the second antenna is decoupled from the first one. In addition, considering the E-field amplitudes in both tables, we can see that the level of the E-field in the proposed design has been improved (from 6.4 to 8.2) in comparison to the initial one.

To determine the effectiveness of the proposed design on the resonance positions, and the radiation characteristics, some important properties are calculated and summarized in Table II for comparison. It is apparent that after using the proposed design, the isolation, the bandwidth, and the gain are considerably improved about $38 \mathrm{~dB}, 5 \%$ and $1.9 \mathrm{~dB}$, respectively. The position of the resonance has changed slightly about $32 \mathrm{MHz}$, which is negligible. The efficiency is almost constant and around 74\%, which is acceptable for a MIMO system.

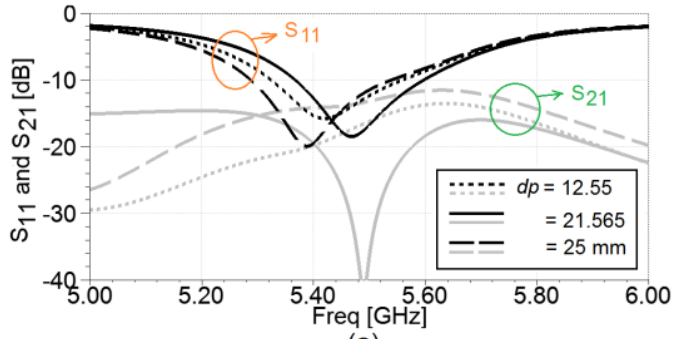

(a)

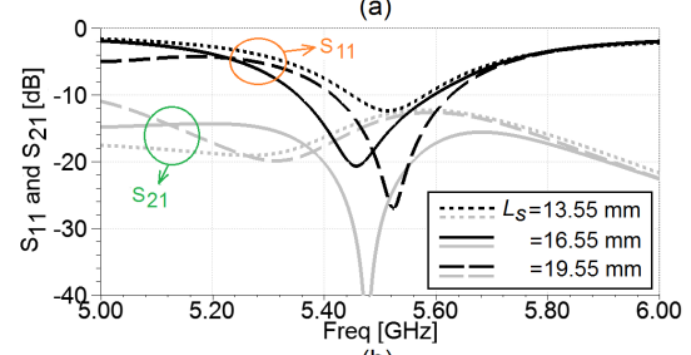

(b)

Fig. 5. Simulated $\mathrm{S}_{11}$ and $\mathrm{S}_{21}$, for different values of (a) $d p$ and (b) $L_{S}$.
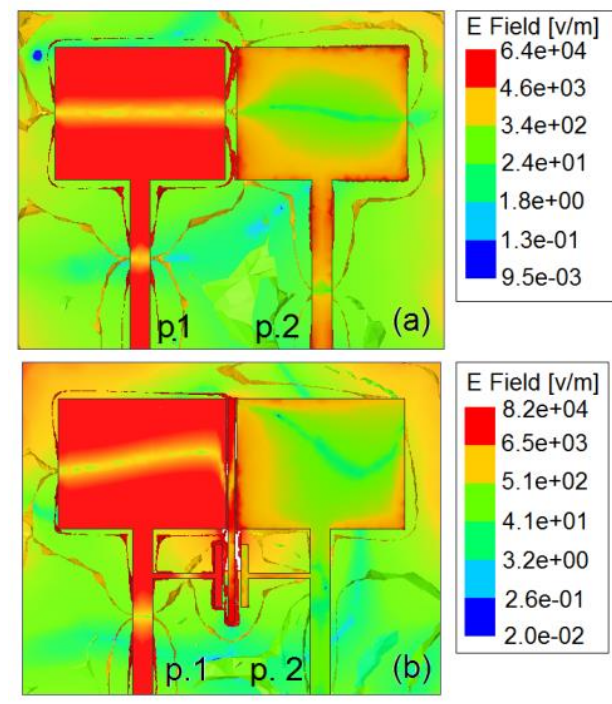

Fig. 6. E Field distributions, (a) before, and (b) after using the proposed isolation structure.

Table II. The impedance and radiation properties of the initial and the proposed designs (when both ports are excited)

\begin{tabular}{|c|c|c|c|c|c|}
\hline Parameters: & Resonance & $\begin{array}{c}\text { Isolation } \\
\left|S_{21}\right|\end{array}$ & $\begin{array}{c}\text { 10-dB } \\
\text { bandwidth }\end{array}$ & Gain & $\begin{array}{c}\text { Radiation } \\
\text { efficiency }\end{array}$ \\
\hline $\begin{array}{c}\text { Initial } \\
\text { design: }\end{array}$ & $\begin{array}{c}5.514 \\
\mathrm{GHz}\end{array}$ & $8.8 \mathrm{~dB}$ & $\begin{array}{c}\text { Not } \\
\text { covered }\end{array}$ & $\begin{array}{c}2.92 \\
\mathrm{~dB}\end{array}$ & $75 \%$ \\
\hline $\begin{array}{c}\text { Proposed } \\
\text { design: }\end{array}$ & $\begin{array}{c}5.482 \\
\mathrm{GHz}\end{array}$ & $46.5 \mathrm{~dB}$ & $5.3 \%$ & $\begin{array}{c}4.79 \\
\mathrm{~dB}\end{array}$ & $73 \%$ \\
\hline
\end{tabular}

\section{IMPLEMENTATION, RESUlTS, DisCUSSION AND COMPARISON}

To validate the design, the optimized array was fabricated and measured. The photographs of the prototype are shown in Fig. 7(a). The measured $S_{11}$ and $S_{21}$ compared to the simulated results are presented in Fig. 7(b). As shown, a better matching (about $23 \mathrm{~dB}$ ) compared to the simulation is provided. In addition, the measured 10$\mathrm{dB}$ bandwidth is about $5.5 \%$ from 5.32 to $5.64 \mathrm{GHz}$ that is close to the simulated result. A good agreement between the simulation and measurement can be observed. The measured isolation (about $50 \mathrm{~dB}$ ) is also better than the simulated one. These improved impedance characteristics guarantee good performance in a MIMO system. 
Table III. Comparison between the proposed design and the other works

\begin{tabular}{|c|c|c|c|c|c|}
\hline Ref. No. & $\begin{array}{c}\text { Resonant } \\
\text { frequency }\end{array}$ & $\begin{array}{c}\text { Edge-to-edge } \\
\text { distance }\end{array}$ & $\begin{array}{c}\text { Mutual coupling } \\
\text { reduction }\end{array}$ & $\begin{array}{c}\text { Design } \\
\text { complexity }\end{array}$ & VIA \\
\hline$[1]$ & $2.45 \mathrm{GHz}$ & $10 \mathrm{~mm}\left(0.08 \lambda_{0}\right)$ & $20.0 \mathrm{~dB}$ & high & yes \\
\hline$[2]$ & $10.0 \mathrm{GHz}$ & $1.8 \mathrm{~mm}\left(0.06 \lambda_{0}\right)$ & $50.0 \mathrm{~dB}$ & low & yes \\
\hline$[8]$ & $5.00 \mathrm{GHz}$ & $2.0 \mathrm{~mm}\left(0.033 \lambda_{0}\right)$ & $34.3 \mathrm{~dB}$ & moderate & no \\
\hline$[11]$ & $2.30 \mathrm{GHz}$ & $20 \mathrm{~mm}\left(0.15 \lambda_{0}\right)$ & $35.0 \mathrm{~dB}$ & moderate & no \\
\hline This work & $5.5 \mathrm{GHz}$ & $1.0 \mathrm{~mm}\left(0.018 \lambda_{0}\right)$ & $44.0 \mathrm{~dB}$ & moderate & no \\
\hline
\end{tabular}

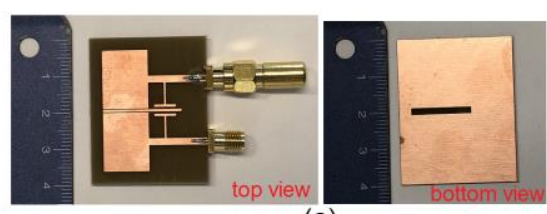

(a)

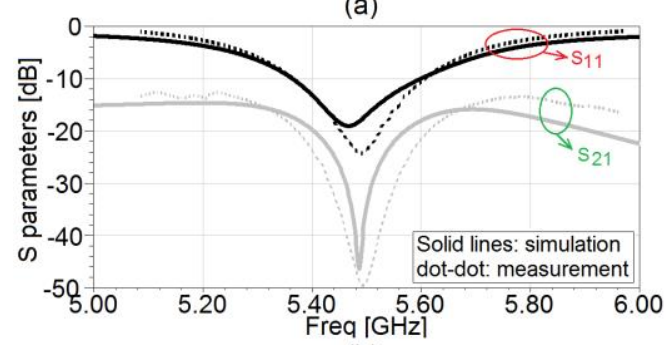

(b)

Fig. 7. (a) The photograph of the prototype and (b) the measured and simulated return losses of the proposed design.

Fig. 8 shows the measured radiation patterns of the initial and the proposed designs. These results indicate that the antenna has broadside radiation patterns with small back lobes and low cross polarization components. As shown, after using the proposed technique, the radiation patterns remain unaffected, especially around the main beam. In addition, the main characteristics, $3 \mathrm{~dB}$ beamwidth, and the maximum gain direction also remain unchanged. Furthermore, a good agreement between the simulated and the measured graphs in both planes $(\mathrm{H}-$ and E-planes) can be observed. A small discrepancy between the simulation and the measurement results is due to the fabrication, soldering and measurement tolerances.

Finally, ECC and DG must be determined, and their values should be within a certain range. The ECC is a measure of antenna radiation diversity. Both radiation fields and $\mathrm{S}$ parameters can calculate this parameter. However, the $S$ parameter approach is not valid for lossy structure. Therefore, it is preferable to determine ECC (i.e. $\rho_{e}$ ) from the far-field radiation patterns $[2,17]$ as given in (1):

$$
\rho_{e}=\frac{\left|\iint_{4 \pi} F_{1}(\theta, \varphi)^{*} \cdot F_{2}(\theta, \varphi) d \Omega\right|^{2}}{\iint_{4 \pi}\left|F_{1}(\theta, \varphi)\right|^{2} d \Omega \times \iint_{4 \pi}\left|F_{2}(\theta, \varphi)\right|^{2} d \Omega}
$$

where $F_{i}(\theta, \varphi)$ is the radiation field of the $i$ th antenna and "." is the Hermitian product. The ECC for the initial and proposed designs are presented in Fig. 9 for comparison. A considerable decrease in ECC is attained after using the isolator. The measured ECC is less than 0.15 around $5.5 \mathrm{GHz}$ that satisfies the criteria of low correlation $\left(\rho_{e}<0.5\right)$ of MIMO. Therefore, a good diversity performance can be expected.

The DG is the amount of improvement obtained from an array system relative to a single antenna, which is calculated by [2]:

$$
D G=10 \sqrt{1-|E C C|^{2}}
$$

According to (2) and the measured ECC, the DG is equal to 9.7 and more than 9.5 around $5.5 \mathrm{GHz}$, which is suitable for a MIMO system.

Finally, the proposed design is compared with the other recentlypublished MIMO designs with improved isolation to determine the advantages of the proposed design. The properties are summarized in Table III. The proposed design has a better mutual-coupling-reduction
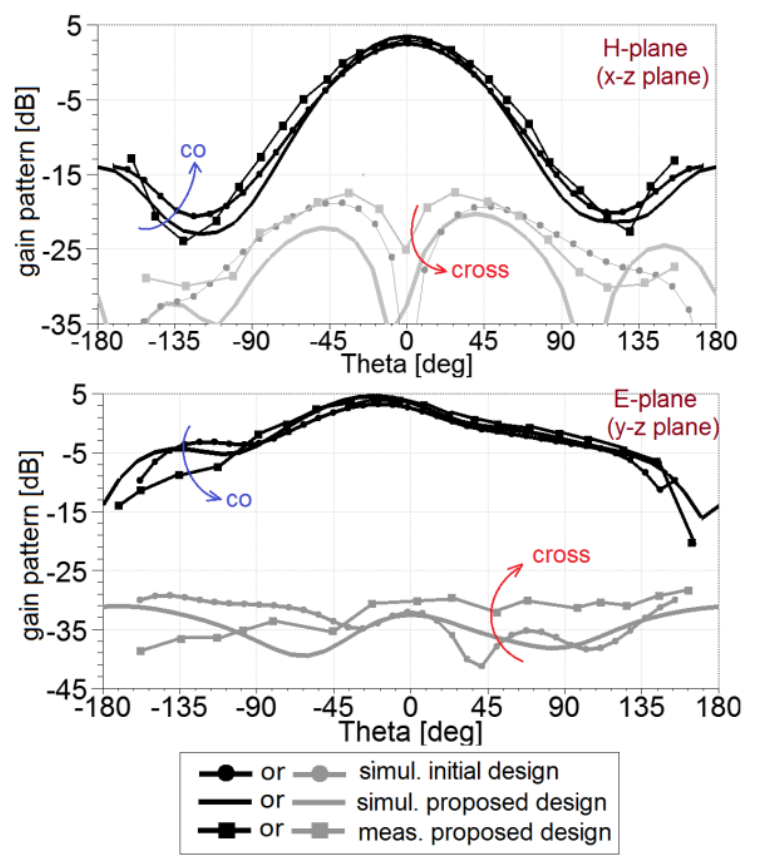

Fig. 8. Simulated and measured radiation patterns.

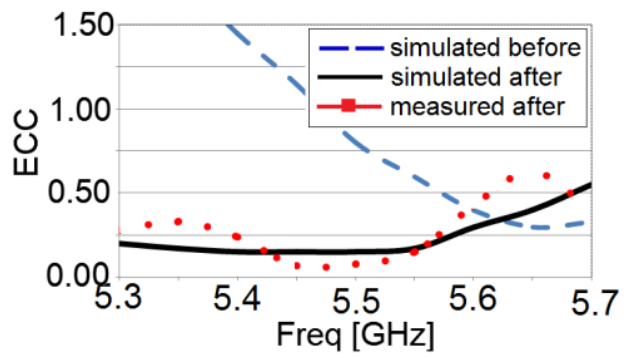

Fig. 9. Simulated and measured ECC of the proposed and initial designs.

than those reported in $[1,8,11]$. Additionally, a tiny distance, less than $0.02 \lambda_{0}$, is selected for the edge-to-edge distance between the antennas which can provide a compact structure. Moreover, the proposed vialess isolator has low design and fabrication complexity in comparison with the others. Finally, the proposed design as a DMN network has a smaller size compared to [18, 19].

\section{CONCLUSION}

A new microstrip-fed array antenna has been proposed for MIMO systems, and shown to be a viable solution to improve the matching and isolation of the array antenna, simultaneously. These useful characteristics have been obtained without using any conventional matching and isolation structure. Consequently, the total size of the antenna array can be minimized. In this work, the distance between the two near edges of the patches has been kept to about $1 \mathrm{~mm}$ (much smaller compared to the other published works). The fabricated prototype has provided isolation and matching about $50 \mathrm{~dB}$ and $25 \mathrm{~dB}$ at $5.5 \mathrm{GHz}$, respectively. The measured ECC and DG have been presented and found to be of the acceptable for MIMO systems. 


\section{REFERENCES}

[1]. K. L. Wu, Ch. Wei, X. Mei, and Z. Y. Zhang, "Array-antenna decoupling surface", IEEE Trans. Antennas Propag., vol. 65, no. 12, pp. 6728-6738, 2017.

[2]. R. Zaker, "Design of a very closely-spaced antenna array with a high reduction of mutual coupling using novel parasitic L-shaped strips," Int. Jour. RF Microwave Computer-Aided Eng., doi: $10.1002 /$ mmce. 21422.

[3]. D. F. Mamedes, et al., "Analysis of impedance matching techniques in tapered microstrip patch Antenna", 2017 SBMO/IEEE MTT-S International Microwave and Optoelectronics Conference (IMOC) 27-30 Aug. 2017.

[4]. R. Zaker, and A. Abdipour, "A very compact ultrawideband printed omnidirectional monopole antenna," IEEE Antennas Wirel. Propag. Lett., vol. 9, pp. 471-473, 2010.

[5]. M. Razavi-Rad, Ch. Ghobadi, J. Nourinia, and R. Zaker, "A small printed ultra-wideband polygon-like wide-slot antenna with a forklike stub," Microwave Jour., vol. 53, no. 3, pp. 118-126, 2010.

[6]. M. Moharram and A. A. Kishk "Decoupling between two asymmetric non-perfectly matched antennas for MIMO applications," 2012 IEEE International Symposium on Antennas and Propagation \&USNC/URSI National Radio Science Meeting, Chicago, July 8-14, 2012.

[7]. M. Moharram and A. A. Kishk "MIMO antennas efficiency measurement using wheeler caps," IEEE Transactions on Antennas and Propagation, vol. 64, no. 3, pp. 1115-1120, 2016.

[8]. S. Hwangbo, H. Y. Yang, and Y. K. Yoon, "Mutual coupling reduction using micro machined complementary meander line slots for a patch array antenna", IEEE Antennas Wirel. Propag. Lett., vol. 16, pp. 1667-1670, 2017.

[9]. X. B. Sun, and M. Y. Cao, "Low mutual coupling antenna array for WLAN application”, Electron. Lett., vol. 53, no. 6, pp. 368-370, 2017.

[10]. K. Wei, J. Li, L. Wang, Z. Xing, and R. Xu, "S-shaped periodic defected ground structures to reduce microstrip antenna array mutual coupling", Electron. Lett., vol. 52, no. 15, pp. 1288-1290, 2016.

[11]. K. Wei, J. Li, L. Wang, Z. Xing, and R. Xu, "Mutual coupling reduction by novel fractal defected ground structure bandgap filter", IEEE Trans. Antennas Propag., vol. 64, no. 10, pp. 4328-4335, 2016.

[12]. Y. F. Cheng, X. Ding, W. Shao, and B. Zh. Wang, "Reduction of mutual coupling between patch antennas using a polarizationconversion isolator", IEEE Antennas Wirel. Propag. Lett., vol. 16, pp. 1257-1260, 2017.

[13]. A. K. Panda, S. Sahu, and R. K. Mishra, "A compact dual-band $2 \times 1$ meta-material inspired MIMO antenna system with high port isolation for LTE and WiMax applications", Int. Jour. RF Microwave Computer-Aided Eng., vol. 27, no. 8, pp. 1-11, 2017.

[14]. K. S. Vishvaksenan, K. Mithra, R. Kalaiarasan, and K. Santhosh-Raj, "Mutual coupling reduction in microstrip patch antenna arrays using parallel coupled-line resonators", IEEE Antennas Wirel. Propag. Lett., vol. 16, pp. 2146-2149, 2017.

[15]. X. B. Sun, and M. Y. Cao, "Mutual coupling reduction in an antenna array by using two parasitic microstrips", Int. J. Electron. Commun. (AEÜ), vol. 74, pp. 1-4, 2017.

[16]. Q. Li, A. P. Feresidis, M. Mavridou, and P. S. Hall, "Miniaturized double-layer EBG structures for broadband mutual coupling reduction between UWB monopoles", IEEE Trans. Antennas Propag., vol. 63, no. 3, pp. 1168-1171, 2015.

[17]. S. Chouhan, D. K. Panda, M. Gupta, and S. Singhal, "Multiport MIMO antennas with mutual coupling reduction techniques for modern wireless transreceive operations: A review", Int. Jour. RF Microwave Computer-Aided Eng., 2017, doi: 10.1002/mmce.21189.

[18]. Y. -F. Cheng, and K. -K. M. Cheng, "A novel dual-band decoupling and matching technique for asymmetric antenna arrays," IEEE Trans. Microw. Theory Techn., vol. 66, no. 5, pp. 2080-2089, May 2018.
[19]. R. -P. Li, P. Wang, Q. Zheng and R. -Zh. Wu, "Compact microstrip decoupling and matching network for two symmetric antennas," Electron. Lett., vol. 51, no. 18, pp. 1396-1398, 2015.

[20]. Y. Li, C.-Y.-D. Sim, Y. Luo, and G. Yang, "12-port 5G massive MIMO antenna array in sub-6 GHz mobile handset for LTE bands 42/43/46 applications," IEEE Access, vol. 6, pp. 344-354, 2017.RF Microwave Computer-Aided Eng., 2017, doi: 10.1002/mmce.21189. 